\title{
A New Approach to Data Authentication in Cloud Storage
}

\author{
V. Abhiram ${ }^{1}$, Srikakolapu NVSSST Murty ${ }^{2}$ \\ ${ }^{1,2}$ Department of Computer Science \& Engineering, Srinivasa Institute of Engineering and Technology, Amalapuram
}

\begin{abstract}
Cloud computing is getting more attention from last decades from the various organization because of its outsourcing facility of data storage using Internet. Therefore Security and privacy became more challenging to secure all these data. It definitely assured so that affective cloud doesn't diversify by affective information which is utilized. The innovative DAC method was expected in the clouds for extra verifications to protect information repository. In this method, cloud checks the attestation about effective sequence rather than to know the clients identification before to place the information in the repository. It was also introduced a scheme by which a valid user can have access to decipher the stored data. It also observed that this scheme is better than the existing centralized method.
\end{abstract}

Keywords: Cloud computing, DAC, Security, Privacy and Decipher

\section{Introduction}

Grid Distributing is the Cloud computing is the usage of figuring credits (machinery \& designing) which is transferred like an authority by a system (ordinarily the cyberspace) [1]. This name originates in distinction to affective regular usage about the haze constructed picture in the act of a consultation being sudden unpredictable base which contains within the structure graphs [2]. Cloud computation depends on local managements which contains information about client, programming and calculation [8]. Distributed computing consists of equipment and programming modules that are made available on the Internet as oversaw outsider administrations [7]. These administrations regularly give access to cutting edge programming applications and top of the line systems of server PCs.

\subsection{Characteristics and Service Models}

The striking qualities of distributed computing considers within chronicle effective explanations given beyond NIST are shown below [3]:

- On-demand self-service: A shopper/customer can singularly procurement registering capacities, for example, server time and system stockpiling, as required consequently without requiring human collaboration with every administration's supplier.

- Broad Network Access: Abilities are accessible over the system and got to through standard instruments that advance utilization by heterogeneous slim or thick customer stages (e.g., cell telephones, tablets, and PDAs).

- Resource Pooling: The supplier's figuring assets are pooled to serve various buyers utilizing a multi-occupant model, with diverse physical and virtual assets progressively doled out and reassigned by interest. Here is an emotion regarding area freedom where the end user doesn't have the major share to maintain authority or data on the careful area which gives assets however they might obtain effective scope to determine area at a larger collection of reflection (For Ex., Country, State or Server Farm). Illustrations of assets covered stockpiling, preparing, memory, system data transfer capacity, and virtual machines.

- Rapid Elasticity: Abilities can be quickly and flexibly provisioned, now and again naturally, to rapidly scale out and quickly discharged to rapidly scale in. To the buyer, the abilities accessible for provisioning regularly seem, by all accounts, to be boundless and can be bought in any amount whenever.

- Measured Service: Cloud frameworks consequently control and enhance asset use by utilizing a metering ability at some level of deliberation suitable to the sort of administration (e.g., stockpiling, handling, transmission capacity, and dynamic client accounts). Asset utilization can be overseen, controlled, and reported giving straightforwardness to both the supplier and purchaser of the used administration.

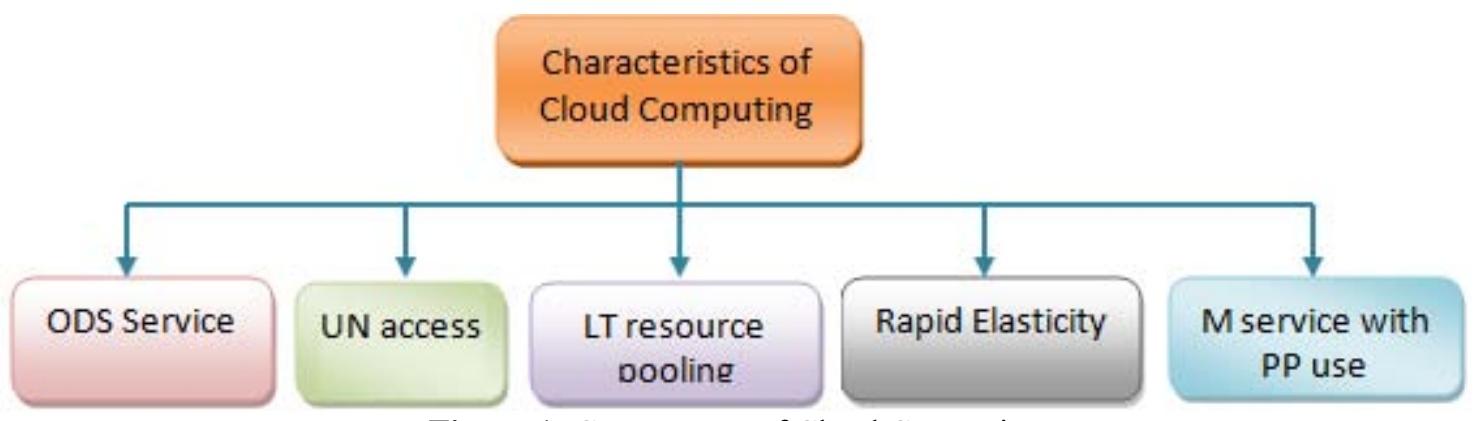

Figure 1: Components of Cloud Computing

Volume 4 Issue 12, December 2015 


\section{International Journal of Science and Research (IJSR) \\ ISSN (Online): 2319-7064 \\ Index Copernicus Value (2013): 6.14 | Impact Factor (2014): 5.611}

\subsection{Maintenance Methods}

Distributed measuring involves 3 diverse administration methods, in general IaaS (Infrastructure as a Service), SaaS (Software as a Service), and PaaS (Platform as a Service). This 3 administration layers or methods were finished by an end client method that exemplifies the end client point of view on cloud administrations. The model is appeared in figure beneath. On the off chance that a cloud client gets to benefits about base seam, being case, user might execute their own procedures depends on assets of grid framework including stay in charge of the bolster, support, and security of these applications herself. On the off chance that she gets to an administration on the application layer, these undertakings are typically dealt with by the cloud administration supplier [4]

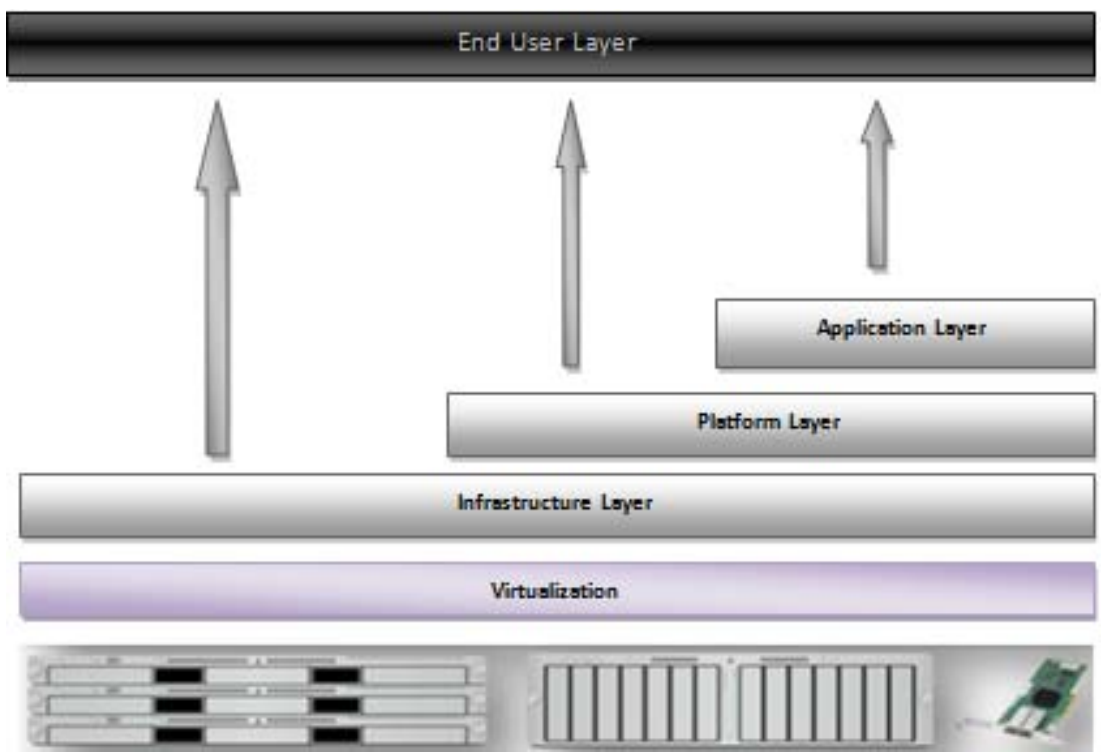

Figure 2: Maintenance Methods Structure.

\subsection{Advantages of Distributed Measuring:}

The following are the advantages of Cloud or distributed computing.

1) Attain recessions of range: Increase Productivity or volume output with some citizens. The price per entity, product or project decreases.

2) Decrease contributing on latest trend framework: Managing simple retrieving about the data with less direct contributing. Depending on the demand pay as your interest to go that is weekly, quarterly or yearly.

3) Globalize your workforce for next to nothing: If there is an network Connection then citizens can easily access the cloud.

4) Streamline Processes: With in less time using few citizen get more work done.

5) Decrease Capital Prices: It's not necessary to spent huge amount on licensing dues, software or hardware.

6) Increase Retrieving: User can retrieve from anywhere and in any time preparing live in a simple manner.

7) Supervise programs further adequately: Complete with in the amount and along with finalization course stages.

8) Limited group coaching is necessary: With a less knowing contour on the software $\&$ hardware problems, the huge tasks on cloud are done by least crowds.

9) Decrease the issuing of innovative software: sweep \& increase beyond the necessity to purchase extravagant spreadsheet licenses or methods.

10)Increase affability: User might divert the way beyond severe "citizens" or "commercial" problems by stave.

\section{Improvements:}

1. Cost: Affective belongings worn are only paid.

2. Surveillance: Grid exponents were segregated within the system beyond other exponents as increased surveillance.

3. Performance: Exponents may be included dynamically to increased achievements. End users have the permissions to all the assets of effective grids crux system.

4. Scalability: Automatically deploy the grid exponents whenever they required.

5. Uptime: Multiple servers are maintained which provides redundancy. When server fails, redundant servers are automatically activated..

6. Control: User can login from any place. Custom instances are downloaded from server software library.

7. Traffic: Additional instances are added to handle the load during extra traffic.

\section{Related Work}

Cloud storage empowers clients to locally stock their information and appreciate affective on-interest top notch cloud working area beyond the weight of neighbourhood equipment and programming administration. In spite of the fact that the advantages are clear, such an administration is likewise surrendering clients' physical ownership of their outsourced information, which unavoidably postures new security dangers toward the information's accuracy in cloud. With a specific end goal is to convey the innovative issue after it accomplish a protected and also reliable distributed 


\section{International Journal of Science and Research (IJSR) \\ ISSN (Online): 2319-7064}

Index Copernicus Value (2013): 6.14 | Impact Factor (2014): 5.611

cache administration, in this paper an adaptable conveyed stockpiling respectability examining component is proposed, using the homomorphic token and disseminated eradication coded information.

The problem of designing the safe explored repository service which is above the opened grid foundation is considered where the authority consumer doesn't trust the end user completely [10]. The developers characterise in an exceptional case, at which some designers who joined late and deviant crypt analysis indigenes with a specific end goal is to complete user goal [9] [11]. Clients review affective benefits which one is the structural engineering will be given to either end users or authority consumers and gives an outline about delayed moves in crypt analysis impelled individually by explored repository [5].

\subsection{Existing System}

- The access control of cloud in existing system is centralized. Remaining mechanisms utilizes ABE which is like entity depends ciphering. This mechanism uses asymmetric key approach and it does not support authentication. The encryptor has the full control over the access rights.

- It gives secured storing attested retrieving limit in grid. Here, users can take a totalized method at which one KDC explores secured locks and entities to all the clients.

\subsection{Drawbacks about the Existed System}

- Use of asymmetric key approach which is slower, costlier, and requires more processing power and use large keys.

- It does not support authentication.

- Large numbers of users are supported in cloud environment to access the cloud and hence it is difficult to maintain them, if the approach is centralized.

\section{Proposed Work}

\subsection{Proposed System}

- In proposed, the control scheme to access the cloud is decentralized which provides security for the data in cloud, that agencies unsigned anonymous attestation.

- Before storing data, grid checks for attestation about series rather than to know the identity of client.

- The scheme allows the authorized clients to deciphering the reserved data which was an added feature.

- The method eliminates redundant problems and assistance generation, changing and reading of information placed within the grid.

\subsection{Benefits of Proposed System}

- Appropriated retrieving limit of information put away in grid such that just approved clients with the substantial properties may get to them.

- Verification of the clients who will place and change their own information about the grid.

- In this verification, affective client's character is shielded from the cloud.

\subsection{Modules}

- System Initialization.

- User Registration.

- KDC setup.

- Attribute generation.

- Sign.

- Verify.

\subsection{Modules Description}

\section{- Initialization of the machine}

Here, first use one prime constant $q$ and also the groups G1 and $\mathrm{G} 2$ that are having a line of $\mathrm{q}$. The team derive the map functioning as

$\wedge_{\theta}: G 1 \times G 1 \rightarrow G 2$. Let $\mathrm{g} 1$ and $\mathrm{g} 2$ be generators of $\mathrm{G} 1$ and $\mathrm{h} \mathrm{j}$ are the producers of $\mathrm{G} 2$, for $\mathrm{j}$ belongs to [tmax], for arbitrary tmax. Let $\mathrm{H}$ be a hash function. Let $A 0=h a 0$ where $a 0 \in Z *$ is chosen at random.

( $T S i g, T V$ e mean TSig is the private key with which a message is signed and TV er is the public key used for verification.

The secret key for the trustee is $T S K=(a 0, T S i g)$ and public key is $T P K=(G 1, G 2, H, g 1, A 0, h 0, h 1 \ldots ., h t m a x, g 2, T V$ er $)$.

\section{- User Registration:}

For a user with identity $\mathrm{Uu}$ the KDC draws at random Kbase $\in G$.

Let $K 0=K 1 / a 0$ base.

The following token $\gamma$ loutput $\gamma=(u$, Kbase, $K 0, \rho)$, where $\rho$ is signature on $u \|$ Kbase using the signing key TSig.

\section{- KDC setup:}

Clouds must decentralize the approach when distributing secret keys and attributes to the users. Clouds can have several KDC's at different locations. We can hav several KDC's and hence the approach is decentralized.

\section{- Generation of an Attribute:}

The TVA verifies the signature contained in using the signature verification key $T V$ in $T P$. This algorithm extracts $K b a$ : from using $(a, j$ from $A S K \mid$ and computes $K_{x}=K 1 /(a+b x)$ base, $x \in J[i, u]$. The

key $K$ can be checked for consistency using algorithm ABS.KeyCheck (TPK, APK $[i], \gamma, K x), \quad$ which checks $\wedge^{\theta}\left(K x, A_{i i} B_{i i}\right)={ }^{e(K b a s e, h j)}$ for all $x \in J[i, u]$ and $j \in[$ tmax $]$.

\section{- Sign:}

The users who can acess the cloud is decided by access policy. The creator of the cloud designs an access policy $\mathrm{Y}$, as a proof of her/his attestation and puts signatures which

\section{Volume 4 Issue 11, November 2015}




\section{International Journal of Science and Research (IJSR) \\ ISSN (Online): 2319-7064}

Index Copernicus Value (2013): 6.14 | Impact Factor (2014): 5.611

indicate the data within the policy. Effective cipher text $\mathrm{C}$ along the $\mathrm{c}$ which was send to grid. The grid checks the signs $c$ and places the data in cloud. Whenever they needs to access affective the text, the grid gives backs the C. If any client have matching entities, he/she can decrypt and access the data.

\section{- Verify:}

During verification, it is time consuming to verify each and every user accessing the cloud. So if the user interested in reading the date which is placed within the grid, the cloud just decrypts it by the keys it receives from the KDC's [6].

\section{Results and Observations}

It gives methodical presentations about methods that are worked are applicable as specified by the technical and business requisites, user manuals and machine thesis.

Framework Verification was Concentrated on the below list of components:

Valid Input: Verified input need to be preferred for described classes.

Invalid Input: The inputs which are not verified are need to be rejected for described classes.

Functions: The described methods need to be practiced.

Output: Relevance external values need to be practiced for described classes.

Systems/Procedures: Consolidated frameworks or methods need to be taken into consideration.

Fig. 3 shows the concept of Centralization and Decentralization.

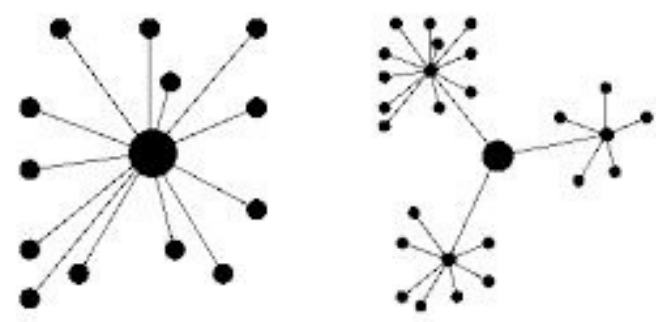

Figure 3: Centralization \& Decentralization

From the above figure centralization is connecting all the nodes to the root node which is placed in the center and the interaction is through the centralized node. Decentralized means the combination of two or more centralized nodes connected to a node. Here, the centralized nodes communicate through the centralized node.

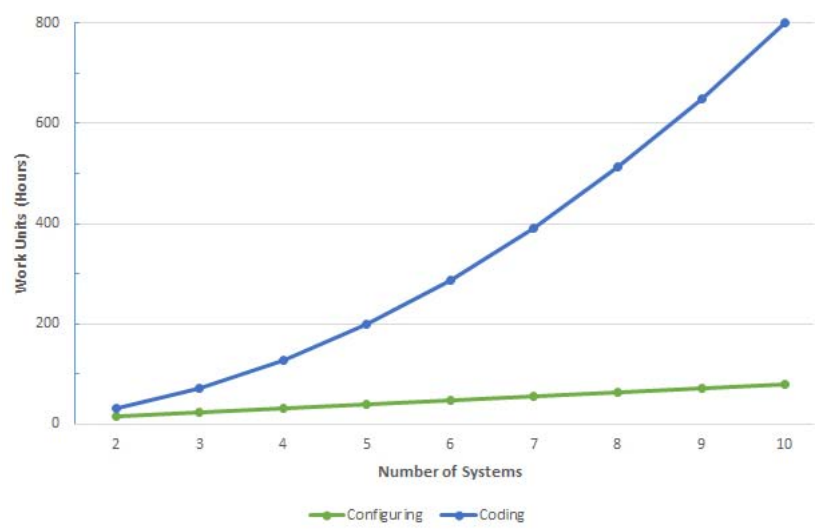

Figure 4: Centralized Vs. Decentralized

\section{Conclusion}

The DAC method was exbhited having unsigned attestation that gives client repeal which avoids repeated attacks. The authorization of affective client who is storing the data is not known to the cloud, but user's credentials are verifies. Keys are distributed by decentralizing the mechanism. Here, one drawback is grid has known affective admittance strategy to every file which is kept within the grid. In upcoming enhancements, authority developers might be interested to cover the entities and retrieved policies of the client.

\section{References}

[1] S. Ruj, M. Stojmenovic, and A. Nayak, "Privacy Preserving Access Control with Authentication for Securing Data in Clouds," Proc. IEEE/ACM Int'l Symp. Cluster, Cloud and Grid Computing, pp. 556-563, 2012.

[2] C. Wang, Q. Wang, K. Ren, N. Cao, and W. Lou, "Toward Secure and Dependable Storage Services in Cloud Computing," IEEE Trans. Services Computing, vol. 5, no. 2, pp. 220-232, Apr.- June 2012.

[3] S. Kamara and K. Lauter, "Cryptographic Cloud Storage.'

[4] R.K.L. Ko, P. Jagadpramana, M. Mowbray, S. Pearson, M. Kirchberg, Q. Liang, and B.S. Lee, "Trustcloud: A Framework for Accountability and Trust in Cloud Computing," HP Technical Report HPL-2011-38, http://www.hpl.hp.com/techreports/2011/HPL-201138.html, 2013.

[5] D.R. Kuhn, E.J. Coyne, and T.R. Weil, "Adding Attributes to Role-Based Access Control," IEEE Computer, vol. 43, no. 6, pp. 79-81, June 2010.

[6] F. Zhao, T. Nishide, and K. Sakurai, "Realizing FineGrained and Flexible Access Control to Outsourced Data with Attribute-Based Cryptosystems," Proc. Seventh Int'l Conf. Information Security Practice and Experience (ISPEC), pp. 83-97, 2011.

[7] H. Li, Y. Dai, L. Tian, and H. Yang, "Identity-Based Authentication for Cloud Computing," Proc. First Int'l Conf. Cloud Computing (CloudCom), pp. 157-166, 2009.

[8] S. Ruj, A. Nayak, and I. Stojmenovic, "DACC: Distributed Access Control in Clouds," Proc. IEEE 10th Int'l Conf. Trust, Security and Privacy in Computing and Communications (TrustCom), 2011. 
[9] S. Jahid, P. Mittal, and N. Borisov, "EASiER: Encryption-Based Access Control in Social Networks with Efficient Revocation," Proc. ACM Symp. Information, Computer and Comm. Security (ASIACCS), 2011.

[10] S. Kamara and K. Lauter, "Cryptographic Cloud Storage."

[11]H. Li, Y. Dai, L. Tian, and H. Yang, "Identity-Based Authentication for Cloud Computing."

\section{Author Profile}

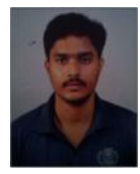

V Abhiram received the Bachelor Degree in Computer Science and Engineering from Swarnandra Engineering College, Narsapur. Now, he is pursuing his M. Tech degree in Computer Science and Engineering from Srinivasa Institute of Engineering and Technology, Amalapuram, India. His research areas are Web Mining, BigData, Cloud Computing.

Srikakolapu NVSSST Murty received his Masters Degree in Computer Science and Engineering from Acharya Nagarjuna University, Guntur. He worked as a lecturer in Degree College from 2002 to 2008 and worked as a lecturer in MCA College from 2008 to 2010 . He has two international Journals and one National Journal. He is now working as an Associate Professor in Srinivasa Institute of Engineering and Technology, Amalapuram, India. His research areas are Wireless Ad Hoc Networks and Mobile Ad Hoc Networks. 